\title{
Integrated Technologies for Smart Buildings and PREdictive Maintenance
}

\author{
Marcello Balzani \\ Fabiana Raco \\ Manlio Montuori
}

\section{Abstract}

The preservation and the regeneration of the existing built heritage is still characterized, even in the context of an increasing digitization of the value and supply chain, by inefficient time and costs management, along the whole life cycle, as well as by discontinuity and lack of information on the one hand, by redundancy and duplication of data on the other. Accessibility, usability and feasibility in order to univocally implement information, also by real-time monitoring, are areas of growing interest to all actors of the building and construction value-chain, with particular reference built heritage knowledge phase, as well as for the stakeholders of complementary industries as ICT, for the development of integrated digital solutions for data acquisition, modeling and visualization.

Keywords

information modeling, digital documentation, information visualisation, built heritage, KETs.

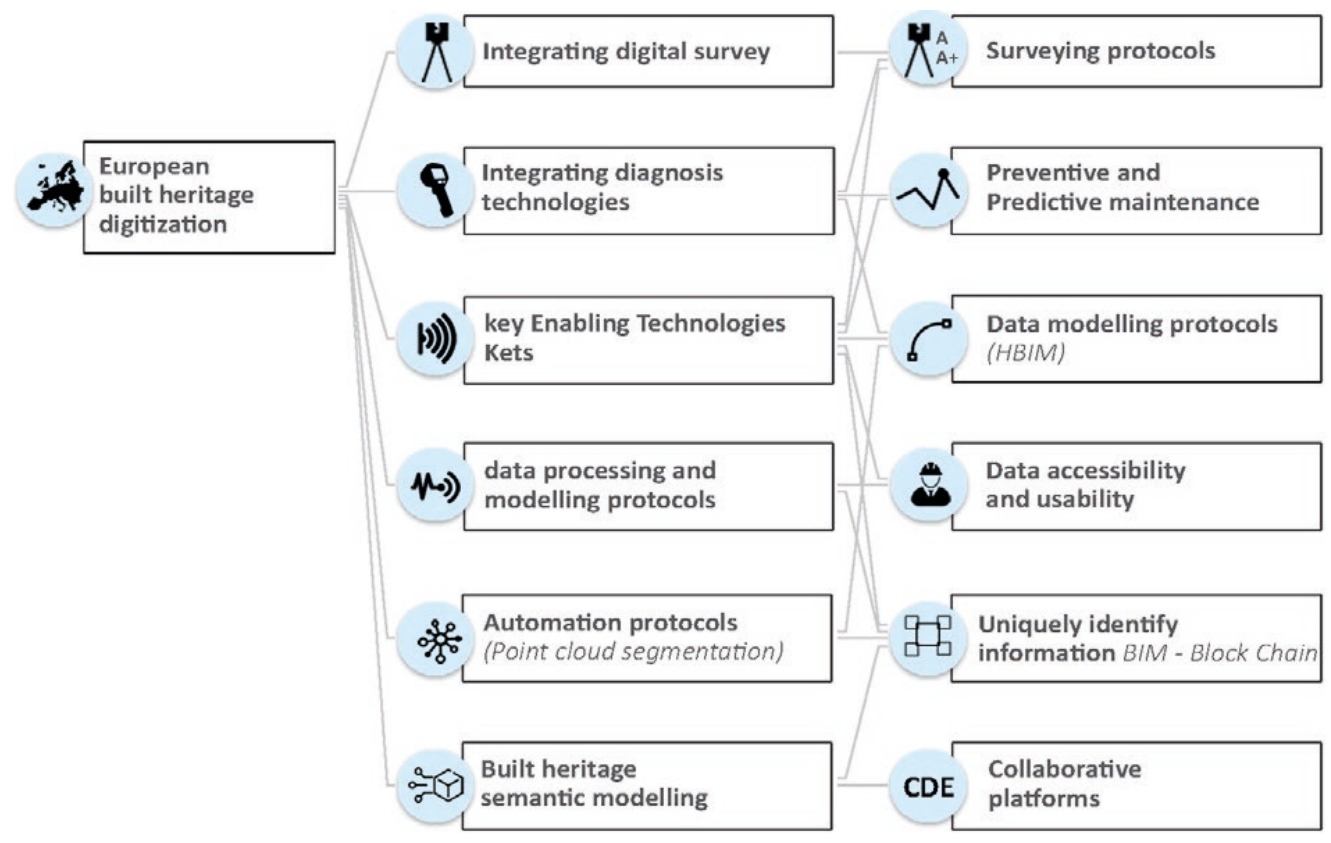




\section{Introduction and Overall Framework}

The InSPiRE project, Integrated technologies for Smart buildings and PREdictive maintenance [I], is funded under the Smart Specialization Strategy of Emilia-Romagna Region, call for "Strategic Industrial Research Projects" 2018 [Hegyi 202 I], and implements the architecture of a predictive diagnostics system for monitoring the state of preservation of materials, components and systems of the existing built heritage that, under normal operating conditions, is approaching the end of its useful life. At the end of the first year of the activity the project is facing several challenging actions aimed at wide spread data integration and sharing, starting from a methodological and technical advancement in built heritage digital documentation. As part of digitization process applied to built heritage as well as to Cultural Heritage, digital documentation and visualisation of the whole building life cycle, from project phase to facility management phase, is emerging as effective strategy in order to support both decision making and sharing of information from different data sources: shape and morphology; diagnostics; safety in use; risk management; maintenance. Implementing collaborative real-time monitoring platforms based on enabling technologies, such as sensor networks, is one of the main goals of the InSPiRE project.

Morever, similarly to the intervention on the cultural heritage, the intervention on the built heritage is characterized by: multiplicity and variety of information sources, with reference to different periods of the building life cycle; lack of homogeneity, and often absence, of organization and hierarchy of information; plurality of design purposes; multiplicity of methods of investigation and technologies applied; plurality of professionals involved [Garzino 20I I].

Fig. I. Project

requirements: multiplicity of data sources characterized the intervention on the built heritage.
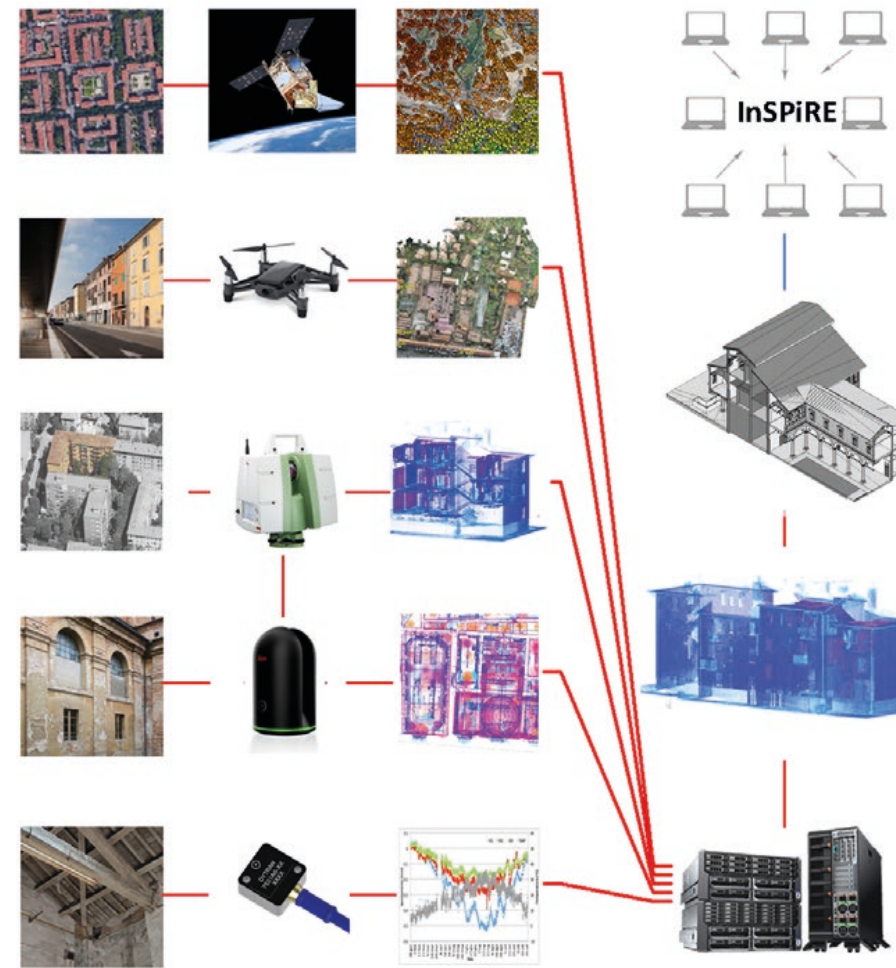

InSPiRE

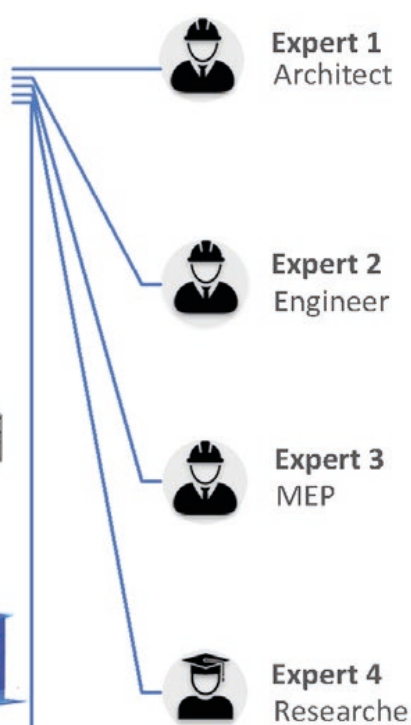

\section{Digital documentation and visualization of the built heritage}

The overall project workflow is developed starting from requirements: what type of data characterized the project on the built heritage (fig. I); what kind of information the variety of professionals involved are asked to provide in order to manage effective decision making processes; with reference to the new ICT technologies applied to the project, such as BIM tools and methods, what kind of both opportunities and boundaries have to be taken into account. Through networks of wireless sensors, based on smartbrick technology, case studies of 
public housing are placed in continuous monitoring in order to: implement monitoring systems based on Integrated Enabling Technologies (KETs); develop predictive algorithms; implement a platform for displaying and managing information, even real time data, supporting awarness during the preservation and the regeneration phases of the existing built heritage.

The sensor network is currently being tested in the context of an ACER case study in Bologna, consisting of a 1950 s block-type residential building.

The acquisition of information from the sensor network and the management of the dataset originated from the diagnostic campaign by multispectral images are currently being implemented within the framework of the above-mentioned case, in order to identify trajectories of behaviour of the building system, in particular in the fields of structural and seismic safety. Starting from previous research results financed within the "Por Fesr Impresa 20 I5" call, the result of the InSPiRE project is a digital platform, currently under development, of strategic decision support for predictive maintenance and management activities that, by implementing intervention procedures on an existing built heritage in borderline and/or emergency conditions, increases its useful life and capitalises its economic value. The involvement of local companies - such as international leaders in the market for the production of materials and systems for the intervention on the existing buildings as well as in the management and processing of big data and in the development of advanced sensors -, which is a strategic action in order to support effective project results, regional and trans-regional cluster industrialization specialisation and value-chain innovation and competitiveness [European Commission 2019], favors the implementation of the specifications of the architecture of the monitoring platform, contributing to the validation and demonstration in the relevant environment (TRL5-6), and to demonstrate the prototypes in the operational environment (TRL7).

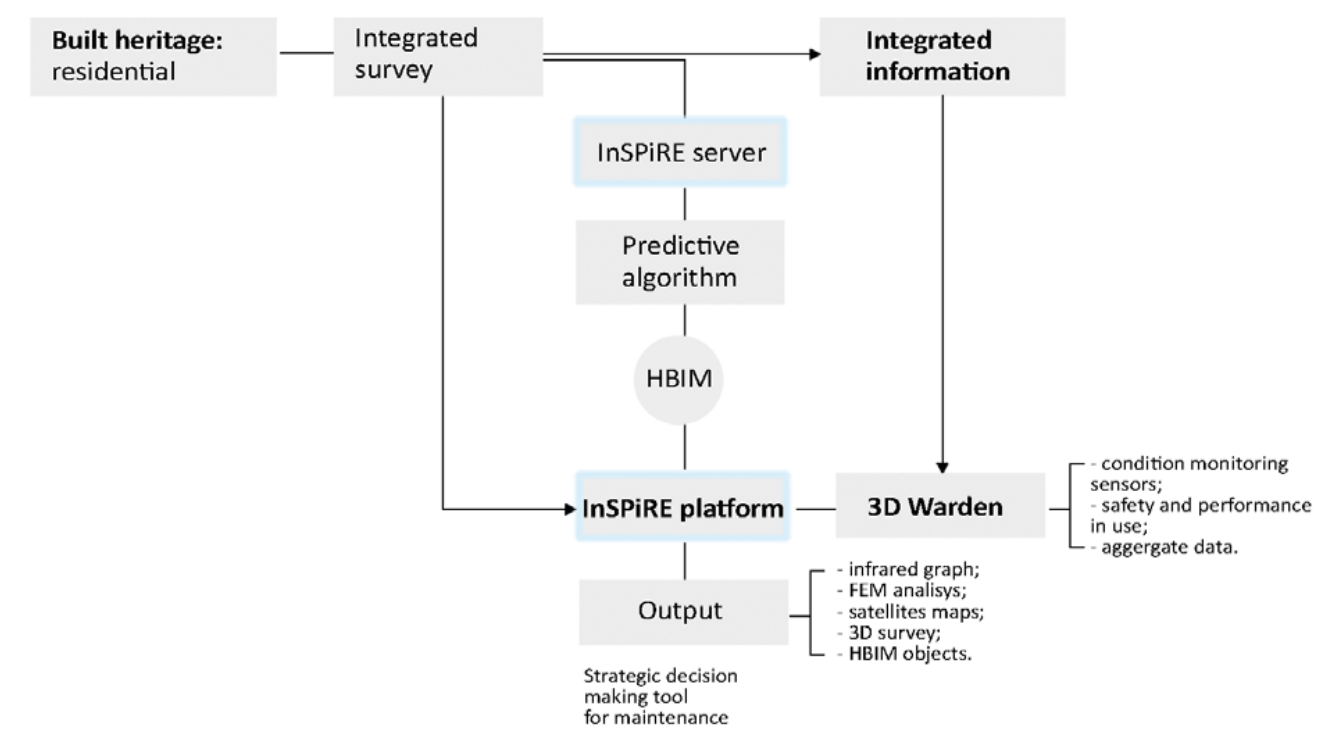

\section{Data Integration and Information Modeling}

The need for the implementation of strategic decision making tools has originated from the fact that there are both a variety of sources of information and methodologies regarding the built heritage documentation.

As a result, the availability of so many active sources providing valuable information to be used by a variety of experts and actors of the value-chain determines whether data redundancy or loss of information when hierarchy, segmentation, accessibility, usability of data as well as built heritage nomenclature are not properly considered.

The starting point in order to implement the InSPiRE digital platform is the data sources hierarchy with reference to the case studies investigated. Each partner deepens, in accor- 
dance with the project objectives, specific skills and pilot tests, a number of different types of source data such as: historical data sets related to the interventions of ordinary and extraordinary maintenance; satellite data for the analysis of the territorial scale; data from continuous monitoring for the analysis of the structural behavior of the building; data from integrated diagnostic campaigns for the evaluation of the energy behavior of the building; data from direct diagnostics for the analysis of mechanical and physical-chemical characteristics of materials in place.

Subsequently, the data modeling phase and definiton of information hierarchy allow to define; trajectories of behavior, for those phenomena that allow an approach to the evaluation also of predictive type; criteria for the subsequent visualization, accessibility and usability of information, with reference to different categories of experts identified as end users of the project results [European Union 20 I8].

\section{Toward Applications and Digital Protocols for Built Heritage Management}

So far, InSPiRE project aims at defining a data integration protocol for built heritage management. From one point of view, the system responds to the request for timely anticipation of the degradation and damage phenomena, by leading the maintenance actions in an adaptive way, with respect to the phenomenology of the degradation cause. Subsequently, the project makes use of the technological skills of industrial ecosystem of the regional territory, whose development can potential benefit from the collaboration during the entire life cycle of the project. Definitely, the development of the different components of the monitoring architecture requires that platform integrates and cooperates with expertise in the field of: restoration of both cultural heritage and built heritage; construction science; digital technologies applied to the survey and diagnostics phases; computer science; chemical and mechanical characterization of the building materials; integrated technologies for building preservation, retrofit and maintenance. The intersectorality and interdisciplinary nature of the project involve strategic sectors and disciplines that respond to the drivers of innovation identified by European Smart Specialization Strategy for the construction industry. The development of technologies for predictive diagnostics applied to facility management is a cross-sectoral challenge to the regional Clust-ER ecosystem, such as Emilia-Romagna industrial and research network, and to the European strategic objectives, relying on enabling technologies (such as new smart materials or the pervasiveness of the Internet of Things technologies) framed, specifically for the Emilia-Romagna Region, in the Clust-ER "Build", "Create", and "GreenTech".

According to the InSPiRE workflow the data integration protocol definition process started studying what and how surveying data to be included in InSPiRE digital platform, as well as analysing how information to be modeled in order to achieve both interoperability and accessibility. Definitely, data visualization is closely related to methodology of archiving and retriving digital information in order to make definition of common topology effective. The platform thus implemented will undergo a testing process by different target end users: professionals; managers of complex real estate assets; maintainers; facility managers.

\section{Conclusions}

Developing integrated digital tools for the management and visualization of information related to the intervention on the built heritage responds to the dual need, expressed by European policies and beyond, to: supporting the adoption of data-driven tools to make decision-making processes more effective, less expensive and more sustainable; promote the industrialization of the supply chain.

Consequently, the objectives that the project pursues are closely related to the diffusion of $\mathrm{H}-\mathrm{BIM}$ protocols for the existing heritage [Hung-Ming 20 I5]. In this sense, the results of InSPiRE are implemented by both objectives and results of other projects Por Fesr funded, such as the eBIM project: existing Building Information Modeling for the management of the intervention on existing heritage. The definition and implementation of semantic 
ontologies in order to organize the knowledge around the complexity of the intervention on the existing heritage cannot be separated so much from the definition of a common lexicon as from the correlation to the purposes of the intervention, rather than to the categories and typologies of the built heritage [Pauwel 20/3].

\section{Notes}

[I] The project "InSpiRE - Integrated technologies for Smart buildings an PREdictive maintenence" involves five partners, including national universities and research centers, such as: Laboratorio TekneHub, Tecnopolo of the University of Ferrara (Lead partner); CIRI EC, Interdepartmental Center for Industrial Research Building and Construction, University of Bologna; CRICT, Interdepartmental Center for Research and for Services in the Construction and Territory Sector of the University of Modena; CNR Istec, Institute of Science and Technology of Ceramic Materials; Flaminia Center for Innovation. Moreover, seven companies are part of the partnership, from the regional territory and beyond, with reference to: production of materials and components for the chain of intervention on the built environment; ICT products; enabling technologies; diagnostic services for the built heritage.

[2] The project "eBIM: existing Building Information Modeling for the management of the intervention on the built heritage", which has received funding from Por Fesr 20 | 4-2020, involves five partners, including national universities and research centers, such as: CIDEA, Interdepartmental Center for Energy and Environment, University of Parma (Lead partner); Laboratorio TekneHub, Tecnopolo of the University of Ferrara (Lead partner): CIRI EC, Interdepartmental Center for Industrial Research Building and Construction, University of Bologna; Centro Ceramico; Certimac. Moreover, ten companies are part of the partnership, from the regional territory and beyond, with reference to: architectural and engineering firm; production of materials and components for the chain of intervention on the built environment; ICT solutions.

\section{References}

European Union (2018). Cultural heritage: Digitisation, online Accessibility and Digital preservation. https://digital-strategy, ec.europa.eu/en/library/european-commission-report-cultural-heritage-digitisation-online-accessibility-and-digital (January $202 \mathrm{I}$ ).

European Commission (2019). Supporting digitalisation of the construction sector and SMEs Including Building Information Modelling. https://ec.europa.eu/docsroom/documents/3828I (April 2020).

Garzino Giorgio, Spallone Roberta, Lo Turco Massimiliano (20l I). Strategie digitali per modelli conoscitivi/Digital strategies for knowledge based models. Garzino Giorgio (ed.). Disegno (E) In_Formazione. Rimini: Maggioli, pp. 70- I I I.

Hegyi Fatime Barbara, Prota Francesco (202I).Assessing Smart Specialisation:Monitoring and Evaluation Systems.https://s3platform. jrc.ec.europa.eu/knowledge-repository? P_P_id=s3ppublications_WAR_s3pcontentsportlet_ INSTANCE_UKfpjUKIJBKt\&P_P_ lifecycle=0\&p_P_state=normal\&p_P_mode=view\&P_P_col_id=column- I\&P_P_col_count= I (March, 202 I).

Hung-Ming Cheng, Yang Wun-Bin, Yen Y-N (20I5). BIM applied in historical building documentation and Refurbishing. In The International Archives of Photogrammetry, Remote Sensing and Spatial Information Sciences. L-5/W7, pp. 85-90.

Lo Turco Massimiliano, Caputo Federico, Fusaro Gabriele (2016). From Integrated Survey to the Parametric Modeling of Degradations. A Feasible Workflow. In loannides Marinos, Fink Eleanor, Moropoulou Antonia, Hagedorn-Saupe Monika, Fresa Antonella, Rajcic Gunnar LiestøIVlatka, Grussenmeyer Pierre (eds.). Digital Heritage. Progress in Cultural Heritage: Documentation, Preservation, and Protection. Cyprus: Springer, pp. 579-589.

Pauwels Pieter, Di Mascio Danilo, De Meyer Ronald, Bod Rens (20I3). Integrating building information modelling and semantic web technologies for the management of built heritage information. In Digital Heritage International Congress (Digital Heritage). Marseille: IEEE, pp. 48I-488.

\section{Authors}

Marcello Balzani, Dept. of Architecture, University of Ferrara, marcello.balzani@unife.it

Fabiana Raco, Dept. of Architecture, University of Ferrara, fabiana.raco@unife.it

Manlio Montuori, Dept. of Architecture, University of Ferrara, manlio.montuori@unife.it 
